\title{
A Physical Origin for Quantum Entanglement and Probabilistic Behaviors
}

\author{
Kenneth H. Schatten 1,2 (1) \\ ${ }^{1}$ NASA/GSFC, Greenbelt, MD, USA \\ ${ }^{2}$ Now at Ai-Solutions, Inc. Lanham, MD, USA \\ Email:kschatten@alum.mit.edu
}

How to cite this paper: Schatten, K.H. (2021) A Physical Origin for Quantum Entanglement and Probabilistic Behaviors. Journal of Modern Physics, 12, 50-58. https://doi.org/10.4236/jmp.2021.121005

Received: August 7, 2020

Accepted: January 11, 2021

Published: January 14, 2021

Copyright (c) 2021 by author(s) and Scientific Research Publishing Inc. This work is licensed under the Creative Commons Attribution International License (CC BY 4.0).

http://creativecommons.org/licenses/by/4.0/

\begin{abstract}
Quantum Mechanics' entanglement and probabilistic behaviors are viewed in the light of Quantum Field Theory's (QFT's) advances made during the last century. In particular, Bohm's version (B-EPR) of the Einstein, Podolsky, Rosen (EPR) experiment is now viewed with the aid of QFT's modern description of electrons. In QFT, free electrons possess a bare core surrounded by a "dressing". The dressing consists of one or more virtual particles/fields pulled from the vacuum during the bound electron's parturition. In QFT, a bound electron's freedom is aided by eliminating its energy losses from bremsstrahlung. The paper develops a "Shimony" numerical model using QFT's free electron structure with the aid of a "random vector paradigm" (RVP). The RVP simply expresses QFT's free electron as a bare core surrounded by an EM dressing. Using this RVP, we imbue newly freed electrons with a vector-like EM spin property of $1 / 2$. From this, the Shimony Monte Carlo computer analysis provides a detailed comparison of the B-EPR experiment as described by Bell. The entanglement property can serve to provide a way to transport shared encoded information. Overall, the electron dressing can convey random elements that may provide QM with its entanglement and probabilistic behaviors.
\end{abstract}

\section{Keywords}

Quantum Mechanics, Entanglement, Probability, Einstein, Electron, Origin, Structure

\section{Introduction}

The background of this research harkens back to the origins of Quantum Mechanics (QM) early in the $20^{\text {th }}$ century. QM's entanglement and probabilistic attributes arose then, and scientists were puzzled by its meaning and behaviors. These views were finalized in a meeting of top QM physicists, using the term: 
"The Copenhagen Interpretation", with the probabilistic aspect meant to describe the quantum wave function, $\psi$, as being a measure of probability, as opposed to a "real" physical property, such as mass density or charge density. Several of the world's best physical theorists at the time, such as Einstein and Schrodinger, objected to this, as probabilities are usually associated with random events, requiring a source of a random element, such as the use of cards or dice. Thus it was upon hearing of this interpretation, Einstein was reported to have declared "God does not play dice with the Universe". Neils Bohr, the founder of the probabilistic interpretation, said: "Einstein, stop telling God what to do". QM also required the existence of another strange phenomenon, called "entanglement". As a result of the Copenhagen meeting, the theoretical viewpoint moved towards the current probabilistic and entanglement viewpoints with a dearth of physical understanding as to why QM exhibits these novel mannerisms.

This paper explores the origin of Quantum Mechanics' 1) behaviors of entanglement, 2) probabilistic as well as, at times yielding a 3) $100 \%$ deterministic response, which may simply be regarded as a probabilistic process of \pm 1 , with negative values indicating probabilities of oppositely oriented spin. These behaviors are displayed through a Monte Carlo computer analysis of the role of electron structure as this affects these three properties. It behooves us to understand how elementary particles may acquire, possess and display these three QM behaviors. To answer this, we need to find a way that seemingly identical electrons can behave individualistically. Namely, consider two electrons: A and B; these two can result in electron A doing one thing and electron B doing another. Hence, seemingly identical electrons need to have specific properties that allow each individual particle to behave individually in accordance with the above three unique attributes. Thus QM displays a puzzling mixture of deterministic, probabilistic and entanglement behaviors.

On the subject of random behaviors, the kinetic theory of gases does have molecules appearing to behave randomly, similar to a stochastic behavior; however, quantum effects are associated with quantization and interference effects totally unlike molecular stochastic behavior. The latter can be understood owing to the huge number of individual molecules involved. Their motions can also be viewed very much as billiard balls classically colliding with one another. The responses are simply associated with the momentum changes and transferring momentum and energy. So, there is a world of difference between classical effects compared with quantum mechanics' digitization associated with its unique discrete quantum jumps plus the plethora of other unexpected behaviors.

One of the early experiments relating to QM's novel entanglement, probabilistic and deterministic behaviors began with the Einstein, Podolsky and Rosen (EPR) gedanken experiment [1]. This term meant that it was a thought experiment, and not conceived to be actualized, as position and momentum are not ideal for observing attributes very accurately. This tackled the problematic condition known as Heisenberg's uncertainty principle, wherein one could not measure both non-commuting properties of a particle, such as momentum and 
position, at the same time! EPR, however, suggested a novel experimental configuration that, in theory, could allow the measurement of non-commuting properties! In its simplest form, two similar or identical particles, 1 and 2, fly off from a common location in opposite directions, thereby acquiring related physical properties. For example, one can measure the position of particle 1, and the momentum of particle 2. Owing to the symmetry of this hypothetical experiment, would allow both particles' non-commuting properties to be simultaneously determined! This method appears to work because the two identical particles serve as each other's mirror image twin. Thereby, one can gain information on both particles non-commuting properties! In Quantum Field Theory (QFT), electrons have structural differences depending upon whether the electrons are bound or free. Lindgren illustrates some of these differences via a QFT equation, shown in Figure 1 [2]. Schatten and Jacobs outlined how virtual photons could play a role in the EPR conundrum [3].

\section{Methods}

We shall be basing our analysis of the electron's structure upon Quantum Field Theory's (QFT) picture of this as described by Lindgren [2]. Figure 2 illustrates

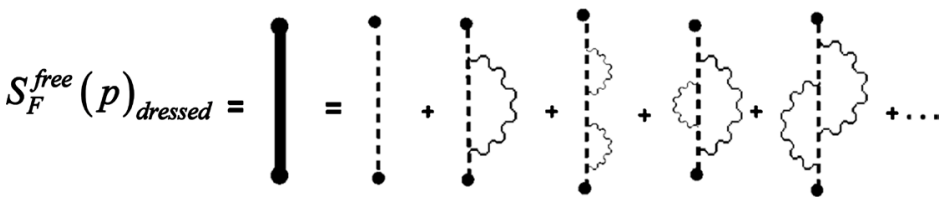

Figure 1. Shows a QFT equation that depicts the difference between a QFT bare electron and a QFT free electron, the latter being a "dressed" electron. A free electron, solid line at left, can be represented as the sum of a bare electron, dashed vertical line, plus its dressing, consisting of one or more virtual particles, shown by dashed semi-circles, towards the right, that the electron can withdraw from the vacuum, corresponding to the remainder of the equation.

\section{QUANTUM FIELD THEORY VIEW OF ELECTRON STRUCTURE}

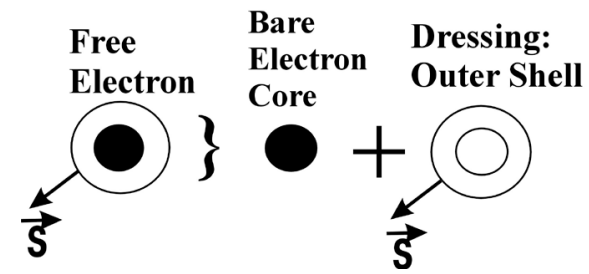

Figure 2. The physical structure of a free electron, corresponding to Figure 1's equation is depicted. On the left the free electron is shown, consisting of a bare core plus an outer shell described in QFT as a "dressing". The bare core may be as small as a point defect in the divergence of the electric field or as large as the classical electron radius. Free electrons contain a dressing of one or more virtual particles drawn from the vacuum, garnered as they leave their bound state. Most commonly this consists of a virtual photon, having spin. When bound in atoms, electrons contain the electron core, plus three specific Pauli spin matrices. These provide several bits of information, little compared with the 3-D vector information associated with the virtual photons acquired into the free electron shell. 
this via a simplified Quantum Field Theory (QFT) physical picture. It helps to bear in mind, that the Maxwell stress tensor consists of tension along the field, and pressure perpendicular to the electric field direction.

For charged particles, virtual photons, shown in the Feynman diagram of Figure 1, are the most common virtual particle. Figure 2 illustrates this via a physical picture that is based on the general Quantum Field Theory (QFT) view. We now consider the details of the Bohm version (B-EPR) of the Einstein, Podolsky, Rosen (EPR) experiment [1] [4]. This offers a more detailed account of free electron properties within the B-EPR experiment than is typically considered. Let us briefly describe how Stern-Gerlach Detectors (SGDs) work. Within these detectors, electrons are attached to neutral silver atoms to create a beam of neutral particles that pass through the SGDs. The SGDs have a strong magnetic field gradient in a direction perpendicular to the beam and detector axis. This field gradient provides a force in the direction of the field gradient, dependent upon the electron's spin in that direction. This separates the particles along the field gradient direction, by amounts dependent upon the spin component or components in that direction. Since electrons have spin $1 / 2$ the beam splits up into two separate beams, spin UP and spin DOWN. This employs a single random vector (RV) via a random vector paradigm (RVP) to model each electron's dressing. For this paper, the usage allows free electrons a greater degree of determinism than the 3 sets of $2 \times 2$ Pauli spin matrices provides for tightly bound electrons. In the B-EPR experiment, the free electrons have their spin gauged by Stern-Gerlach (SG) detectors.

The free electron and positron, in Quantum Field Theory, contain one or more virtual particles in their dressings. These are typically virtual photons, drawn from the vacuum, serving to cloak these particles as they leave any strongly bound state. Like an evanescent electromagnetic field within a conductor, the field gradually diminishes with distance, inhibiting EM radiation. For the B-EPR experiment, the positron and electron leave their common zero-spin state, shown in Figure 3. For the purposes of the EPR experiment, when attached to a neutral atom, these particles behave similarly, allowing Stern-Gerlach Detectors (SGD) to sample their spin attributes. The only pre-condition set for the particles' spins is that the two particles contain oppositely oriented spin vectors, owing to the conservation rule associated with their zero-spin state origin, namely:

$$
\overrightarrow{\boldsymbol{s}}_{E}=-\overrightarrow{\boldsymbol{s}}_{P} .
$$

Equation (2) is, essentially, an "entanglement" equation, creating a bond or commonality that defines the two particles' co-existence, much as identical twins possess. We can refer to this as the B-EPR "spin entanglement equation". Other types of entanglement are also possible, dealing with a property that one system or particle possesses, having an attribute related to another's property. These generally may be considered as being the result of quantum "entanglement", a quantum condition that links the entities together, such as position and momentum, much as EPR originally considered. Namely, one particle can have its 


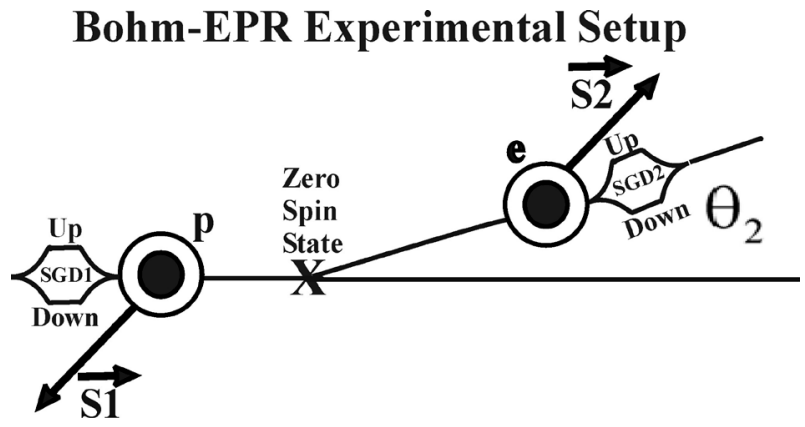

Figure 3. In the B-EPR experiment, a positron, p, particle 1, and an electron, e, particle 2, emanate from a Zero-Spin State located at $\mathbf{X}$. The figure shows the particles' inherent structure, as having a bare core, surrounding which is a "dressing" containing one or more virtual particles (commonly a virtual photon). The two charged fermions: a positron, $\mathrm{P}=$ particle 1 , and an electron, $\mathrm{E}=$ particle 2, exit their parturition site at $\mathrm{X}$. Subsequently, they travel towards the Stern-Gerlach Detectors (see Feynman et al.) SGD1 and SGD2 [5]. These are oriented at relative angle, $\theta_{2}$, with respect to each other. In the Random Vector Paradigm (RVP), each dressing contains an equal and opposite 3D random vector that provides connected spin information: $\overrightarrow{\boldsymbol{s}}_{E}=-\overrightarrow{\boldsymbol{s}}_{P}$, thereby creating a mathematical dependency, appearing as "quantum entanglement."

position measured, and its twin have its momentum measured, and thus obtain both properties for both particles simultaneously! Not often appreciated, is that free electrons can convey vector information in their dressings. The question of the locality of the information remains. In the B-EPR experiment, the particles appear non-locally related, owing to the distant locations from each other, when the two particles' spins are measured. This is an unfortunate conclusion, because the two particles originate from the common zero spin state at $\mathbf{X}$; hence they are locally related.

At this point QFT's role ends, having supplied the dressed electron paradigm. From this, we hypothesized the sum of each particle's dressing can be expressed as a spin vector, which we refer to as the random vector paradigm (RVP), thereby allowing these fermions to possess $3 \mathrm{D}$ random vector spin information, which contains a significantly greater amount of information than is available from tightly bound electrons within an atom.

\section{Results}

To obtain the results, a computer generated simulation of the EPR experiment was carried out. Commonly, this is referred to as a "Monte Carlo" simulation. This term is chosen in particle physics because progress is made through numerical simulations of theory, using dice-like random number generations, rather than theoretical models to compare with experimental data. We use the random vector paradigm, RVP, to generate a Monte Carlo simulation, named Shimony, owing to his exuberance in spreading the interesting paradoxical aspects of the Bohm-EPR experiment. It is through our Monte Carlo simulation that we obtain our numerical results. For each point in the figure, 500 individual Bohm-EPR simulated electrons were passed through the numerical code, and the 


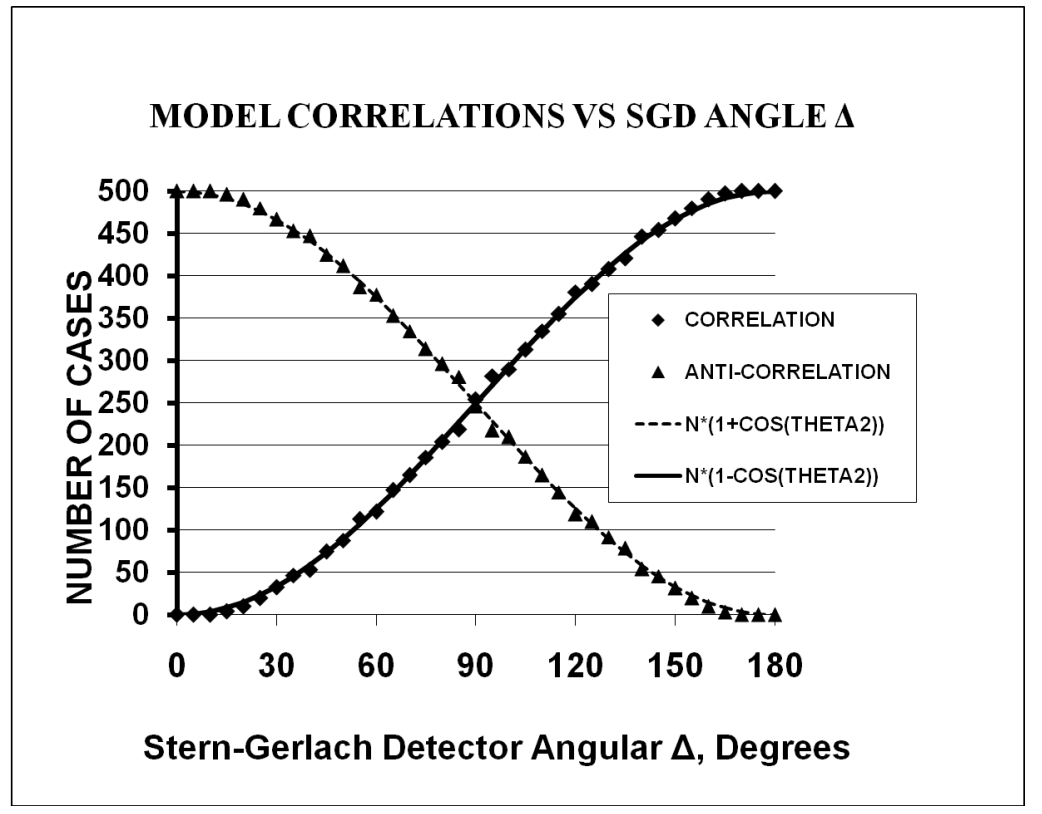

Figure 4. Correlations and Anti-Correlations in the B-EPR experiment (diamond and triangle points), compared with Bell's formulae (the solid and dashed curves). Positive correlation indicates the SGDs read ++ or -- . Anti-correlation indicates opposite responses +- or -+ . The abscissa indicates the relative angular orientation between the two Stern-Gerlach detectors. At an angle of $0^{\circ}$ anti-correlations dominate; at $180^{\circ}$ positive correlations exist; whereas at $90^{\circ}$, stochastic behavior ensues: a puzzling tapestry of responses. Although there seems to be general agreement, these results only apply to a single EPR experimental setup. There are many other puzzling EPR experiments as well as non-EPR experiments.

results summed. For the case of positive correlations, each point added meant that either $\mathrm{a}++$ or -- spin orientation was detected by the two Stern-Gerlach Detectors (SGD). Each anti-correlation point meant that either a +- or a -+ SGD1 and SGD2 detector results occurred and then the sums were added up.

Figure 4, obtained from the Shimony algorithm, shows the correlations and anti-correlations in the model simulation, as the triangle and diamond sums, compared with Bell's formulae shown by the solid and dashed curves.

\section{Discussion}

The numerical correlations and anti-correlations compare well with Bell's summary of the B-EPR experimental form. Bell [6] found "non-local hidden variables" were involved to explain his findings. Despite our results being in accord with Bell's, we draw a somewhat different conclusion. We can agree with Bell's calling the spin properties of the two particles "hidden variables". We consider that the $3 \mathrm{D}$ spin vectors obtained by the free positron-electron pairs used in the experiment go beyond the normal atomic Pauli spin matrices. There is a difference in our understanding of the cause of the correlations found, however.

We agree that the two fermions cannot affect each other, owing to their separation, except "non-locally". Traditionally, locality in physics has been taken to indicate that an object is only influenced by local forces or particles, etc., as op- 
posed to distant effects, often called "action at a distance". Still, our understanding of the experiment, is that it starts out at the same event, let us say, at $\mathrm{T}_{0}$, when the positron electron pair were released from the Zero-Spin-State towards two different directions within the experiment, namely towards the two detectors. With the two particles having "entangled" spin vectors, the detectors each measure their respective spin vector "locally", associated with the interaction of the particle as it is observed by the detector through it locally sampling its spin vector.

It appears that non-locality in quantum mechanics has been accepted, as a strange quantum phenomenon that seemingly violates the special theory of relativity through the "spooky action at a distance". Essentially, the physics community has given a green light to non-locality in quantum mechanics, since everything else in QM is also so puzzling. In the B-EPR experiment, it is possible to observe particles 1 and 2 close enough in time, so that they have a spatial separation, not a temporal one. Hence, the two-particle observations appear not to be causally related, namely with a space-like interval between the two observations, one makes the assumption that neither can affect the other by direct influences. Then, when we see the observations are surprisingly correlated, this is taken to mean there is "spooky action at a distance", or non-local influences, or other words to that effect. It appears to this author, that since the experiment began at a time, say $\mathrm{T}_{0}$, when the Zero-Spin-State released the two fermions, and later, both observations were temporally related to this time, $\mathrm{T}_{0}$. As a result the physics going on is best considered local, rather than non-local! One can easily fool ourselves into thinking that the measurement on one is affecting the other, whereas in reality, the properties of both were "caused" earlier at time, $\mathrm{T}_{0}$. A correlation is NOT the same as causality. The data are correlated because they both arose from a common zero-spin-state, but neither one "causes" the other. They are simply mathematically linked, both "caused" by an event that branded both, earlier in time than either was observed, namely $\mathrm{T}_{0}$. Hence both observations are "time-like" events associated with their common earlier origin at $\mathrm{T}_{0}$. In this view, there appears to be a total absence of any "non-local, action at a distance, spooky" behaviors occurring within the B-EPR experiment.

This is not to say that the experiment is not a brilliant one, nor that further information cannot be gleaned by more sophisticated EPR and non-EPR experiments that may shed light on the correlations found, with further room for inventive geometries, timings, etc. Nevertheless, it does seem that, at least for the standard B-EPR experiment that Bell reported on, the observations seem to reveal valuable, interesting correlation forms that can really shed a great deal of light on how Quantum entanglement and probabilistic behaviors arise. Quantum Mechanical behaviors are really extraordinarily difficult to fathom, and most, particularly the B-EPR behaviors indeed are mostly counter intuitive. But the beautiful aspect of Quantum Mechanics, is that if one follows a good prescription, things do manage to get sorted out properly. 
Beyond the results found here, there exists a panoply of other EPR-type experiments, as well as other non-EPR quantum puzzles that have totally different behaviors than the B-EPR one described here. Bohm referred to the QM puzzles with his viewpoint called "the Implicate Order", indicating some larger-scale order exists amidst the chaotic nature involved within these tiny bits of matter [4]. It is difficult to understand what David Bohm meant with this term. Yet he spent his lifetime considering quantum mechanics, and certainly entanglement is a major quantum component requiring understanding. To this author, it may indicate that he actually believed in determinism within physics much as Einstein did, however, probabilistic behaviors arise simply as a reflection of our inability to completely know all the "hidden variables" involved. With weather and climate forecasting, we now know that many systems behave "chaotically", not meaning a disparaging aspect of their behavior, but simply, in the mathematical sense, as a "sensitivity to initial conditions". None of this was known during most of the $20^{\text {th }}$ century, so quantum's entanglement and probabilistic behaviors were doubly perplexing then. We now recognize that there are mathematical/physical limits beyond which one can never see into the future.

I shall add a few further thoughts about Quantum entanglement. It appears that entanglement is an immensely powerful controlling process, much as a rigid bridge can span distances and thereby reduce travel time. This is how the spin orientations of the two particles in the B-EPR experiment remain connected, despite even should vast distances separate the particles. Yet we also know that the two particles behave within the constraints of special relativity. However, because of the constraints that Quantum Mechanics imposes on particles, these connections have been suggested for a wide variety of uses in various diverse fields, such as computers, cybernetics, data encryption, theoretical physics, and many others. The current findings do NOT take away from the many possible ways that quantum phenomena can play in advanced computer systems, such as using Qubits, etc. Rather, the reverse is true, these experiments actually point more strongly to the fact that quantum correlations are powerful and can lead to $100 \%$ determinism if undertaken properly. Hence there are ways of achieving advanced data processing, well beyond the standard computer bits of 0 and 1. In quantum computers, using Qubits, the phases of the electromagnetic field can greatly expand the processing power using phase information, rather than just the limiting 1 standard bit of present day computers. This is a wide open field. Additionally, now that we may better understand how quantum entanglement works, there may be greater confidence in using these methods, particularly in encryption, data security, and simple data processing to enhance forecasting with greater accurate models.

\section{Acknowledgements}

The author was fortunate to have met Abner Shimony, who generously shared 
his enlightened knowledge and deep understanding of many quantum puzzles, including the Bohm-Einstein, Podolsky, and Rosen experiment. The author also appreciates discussions with Sabatino Sofia, Verne Jacobs, and Hans Mayr.

\section{Author Information}

The author works for ai-Solutions, Inc., Lanham, MD USA.

\section{Conflicts of Interest}

The author declares no conflicts of interest regarding the publication of this paper.

\section{References}

[1] Einstein, A., Podolsky, B. and Rosen, N. (1935) Physical Review, 47, 777-780. https://doi.org/10.1103/PhysRev.47.777

[2] Lindgren, I., et al. (1998) Physical Review A, 58, 1001-1015. https://doi.org/10.1103/PhysRevA.58.1001

[3] Schatten, K. and Jacobs, V. (2020) Canadian Journal of Physics, 98, 895-899. https://doi.org/10.1139/cjp-2019-0537

[4] Bohm, D. (1951) Quantum Theory. Prentice Hall, Englewood Cliffs.

[5] Feynman, R.F., Leighton, R.B. and Sands, M. (1965) Feynman Lecture Notes, II 35.1-6, III 6.1-6.14. Addison-Wesley, Reading.

[6] Bell, J.S. (1966) Reviews of Modern Physics, 38, 447-452. https://doi.org/10.1103/RevModPhys.38.447 